Bull. Mater. Sci., Vol. 36, No. 4, August 2013, pp. 751-754. (C) Indian Academy of Sciences.

\title{
Magnetic resonance in superparamagnetic zinc ferrite
}

\author{
JITENDRA PAL SINGH* , GAGAN DIXIT ${ }^{\dagger}$, R C SRIVASTAVA $^{\dagger \dagger}$, HEMANT KUMAR ${ }^{\dagger \dagger}$, \\ H M AGRAWAL ${ }^{\dagger \dagger}$ and PREM CHAND \\ Department of Applied Science, Krishna College of Engineering, Ghaziabad 201 007, India \\ ${ }^{\dagger}$ Materials Science Division, Inter University Accelerator Centre, New Delhi 110 067, India \\ ${ }^{\dagger}$ Department of Physics, Govind Ballabh Pant University of Agriculture and Technology, Pantnagar 263 145, India \\ ${ }^{\sharp}$ Indian Institute of Technology, Kanpur 208 016, India
}

MS received 5 January 2012; revised 9 September 2012

\begin{abstract}
In the present work, we have synthesized zinc ferrite nanoparticles by nitrate method. Presence of almost zero value of coercivity and remanence in the hysteresis of these samples shows the superparamagnetic nature at room temperature. Electron paramagnetic resonance spectroscopy performed on these samples in the temperature range 120-300 $\mathrm{K}$ indicates the systematic variation of the line-shapes of the spectra with temperature. Both $g$ value and peak-to-peak linewidth decrease with increase in temperature. The variation of $g$-values and peak-topeak linewidth with temperature has been fitted with existing models and we observed different values of activation energies of the spins for both the samples.
\end{abstract}

Keywords. Nanoparticles; magnetic materials; electron paramagnetic resonance spectroscopy.

\section{Introduction}

It is well established that bulk zinc ferrite is an antiferromagnetic material having a Néel temperature of $10 \mathrm{~K}$. It exhibits normal spinel structure i.e. all the $\mathrm{Zn}^{2+}$ remain at $A$-site (tetrahedral) and all the $\mathrm{Fe}^{3+}$ remain at $B$-site (octahedral) (Cullity 1971). This material has become a material of great attention for scientific community from last two decades due to its extremely different magnetic behaviour in nanoregime compared to its bulk counterpart (Roy et al 2006; Xue et al 2007; Singh et al 2008a, b; Sultan and Singh 2010). Nanosized zinc ferrite shows superparamagnetism at room temperature followed by its mixed spinel structure. The magnitude of magnetization and degree of cation inversion are affected by the particle size in nanoregime. In this context, various authors have investigated the size dependent properties of nanosized zinc ferrite and observed increment in cation inversion with decrease in particle size resulting in enhancement in magnetization for smaller sized systems (Roy et al 2006; Xue et al 2007; Singh et al 2008a, b; Sultan and Singh 2010). Apart from particle size, other properties are very much sensitive to the method of syntheses (Sato et al 1990; Jaydevan et al 1994; Ahn et al 2002; Yu et al 2003; Hofmann et al 2004; Shenoy et al 2004; Nakashima et al 2007; Azam et al 2009). Mechanism of magnetization in the system was studied by various authors who observed that magnetization in this system cannot only be explained by superparamagnetism but it may have ferrimagnetism, simultaneous existence of ferri and antiferromagnetism, ferromagnetism and spin-glass state (Chinnasamy et al 2000; Lee et al 2002; Shim et al

\footnotetext{
*Author for correspondence (jitendra_singh2029@rediffmail.com)
}

2006; Yao et al 2007). It has also been observed that not only the magnitude of magnetization but also the nature of magnetic ordering depends on the method of preparation (Singh et al 2012).

To establish the mechanism of magnetization in nanosized zinc ferrite synthesized by chemical route, we have done Mössbauer, electron paramagnetic resonance spectroscopy (EPR) followed by vibrating sample magnetometer measurement. The synthesized system exhibits superparamagnetism as investigated at room temperature by Mössbauer and EPR spectroscopy (Singh et al 2008a, b, 2010). In-field Mössbauer spectroscopy at low temperature performed on these samples indicate that nanosized zinc ferrite exhibits antiferromagnetic ordering below blocking temperature (Singh et al 2012). To get information about the spindynamics of nanosized system, EPR spectra was recorded at high temperature ranging from $300-850 \mathrm{~K}$. It was investigated that responsible phenomena for relaxation mechanism is not only governed by direct and orbach process but some other exchange processes are also affecting this mechanism and are discussed in detail elsewhere (Singh et al 2009). Also these samples indicate different variations of $g$-value and peak-to-peak linewidth with temperature, which depends upon the particle size. In the present work, we have further extended EPR study at low temperature (120-300 K) for similar particle size in order to investigate spin dynamics in superparamagnetic zinc ferrite at low temperature.

\section{Experimental}

Zinc ferrite nanoparticles were synthesized by nitrate route annealing, the precursor at 300 and $500{ }^{\circ} \mathrm{C}$ (Singh et al 
2008a, b). The crystalline phase and structure were studied with the help of X-ray diffractometer using $\mathrm{CuK} \alpha$ radiation. The morphology and size of these nanoparticles were estimated from high resolution transmission electron microscopy (HRTEM). Room temperature hysteresis for all the samples was recorded by using a Princeton-109 vibrating sample magnetometer. Further, EPR spectra for both the samples were recorded in the temperature range 120-300 K by Bruker-EMX EPR spectrometer.

\section{Results and discussion}

Synthesized samples exhibit pure spinel phase and the crystallite size of these samples are 13 and $19 \mathrm{~nm}$ corresponding to sintering temperatures 300 and $500^{\circ} \mathrm{C}$, respectively. These samples are coded as ZF13 and ZF19 and detailed analysis of their structural and morphological properties have been published (Singh et al 2013). Figure 1 shows room temperature hysteresis for the samples ZF13 and ZF19. Almost zero value of coercivity and remanance in these nanoparticles show the presence of superparamagnetism. The higher value of magnetization is observed for sample ZF13 compared to ZF19 and is relevant with other studies of nanosized zinc ferrite (Chinnasamy et al 2000; Lee et al 2002; Roy et al 2006; Shim et al 2006; Yao et al 2007; Singh et al 2012).

Figure 2 shows X-band EPR spectra of the samples at different temperatures in the range $120-300 \mathrm{~K}$. A visual inspection of EPR spectra shows that samples ZF13 and ZF19 have one moderate resonance signal at all temperatures. We have estimated $g$-value from EPR spectra by using the formula (Poole and Farach 1971):

$$
g=\frac{h v}{\beta H_{\mathrm{r}}},
$$

where $H_{\mathrm{r}}$ is the resonance field, $v$ the frequency and $\beta$ the Bohr magneton. For both the samples, $g$-value $(\sim 2.253 \pm$

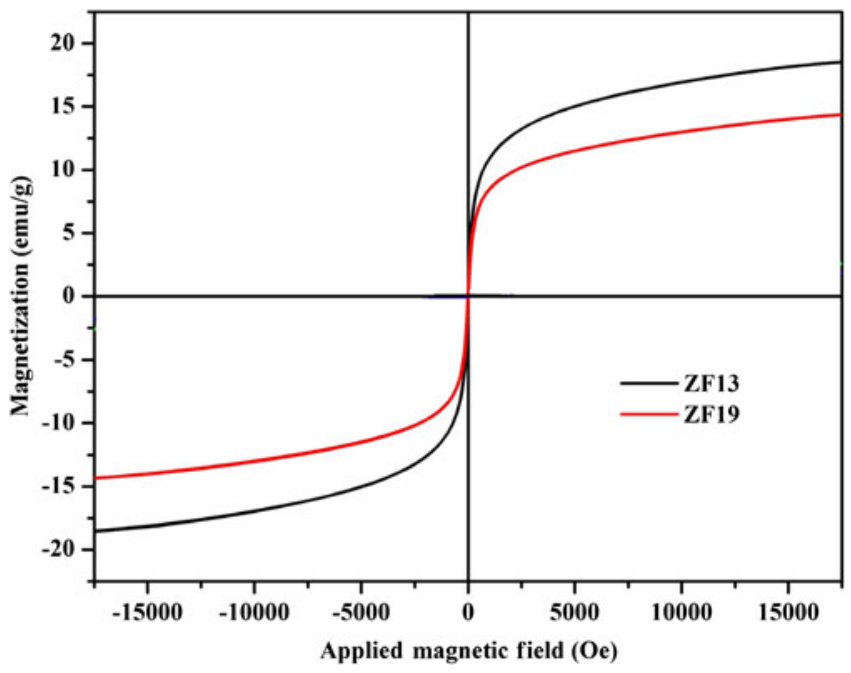

Figure 1. Hysteresis curves of samples ZF13 and ZF19 at room temperature.
0.006 ) at $120 \mathrm{~K}$ is almost same within the experimental error but at room temperature ZF19 has smaller $g$-value compared to ZF13 (table 1). It is clear from this table that $g$-values decrease with increase in temperature for both the samples. Similar behaviour of $g$-values with temperature has also been reported for other ferrite systems (Singh et al 1993; Wu et al 2004; Dixit et al 2012) and may be attributed to the effect of reduction in magnetic ordering with temperature. In the present work, $g$-values are slightly higher compared to that obtained from our previously synthesized zinc ferrite system (Singh et al 2008a, b).

The values of peak-to-peak linewidth $\left(\Delta H_{\mathrm{PP}}\right)$ of samples ZF13 and ZF19 are $1219 \pm 16$ and $1294 \pm 16$ Oe, respectively at $120 \mathrm{~K}$. Both samples exhibit decrease in the value of $\Delta H_{\mathrm{PP}}$ with increase in temperature and attain almost same value of $\sim 649 \pm 16$ and $645 \pm 16$ Oe at room temperature (table 1). The linewidth of EPR signal for any ferrite material generally originated from two sources: (i) magnetic dipole interactions among particles and (ii) superexchange interactions between magnetic ions through oxygen ions. Linewidth may get broadened or narrowed depending upon the interaction inside the material. Dominant dipole interactions give rise to large linewidth and $g$-factor, however, small linewidth and lower $g$-values are observed, where superexchange interactions are dominant (Li et al 2000). Higher value of $\Delta H_{\mathrm{PP}}$ at low temperature shows dominancy of dipolar interaction among the particles. This result is in agreement with EPR study performed on nickel ferrite nanoparticles (Dixit et al 2012), however, occurrence of narrow peakto-peak linewidth at room temperature in these samples may be due to dominant superexchange interaction. The increased temperature will provide extra thermal energy to the atoms, thereby increasing motion of the ions. This causes stronger superexchange interaction among the cations through oxygen ions, consequently, $\Delta H_{\mathrm{PP}}$ and $g$-factor decrease with increase in temperature (Singh et al 1993). Temperature variation of linewidth and $g$-values of Ni-Zn ferrite has been

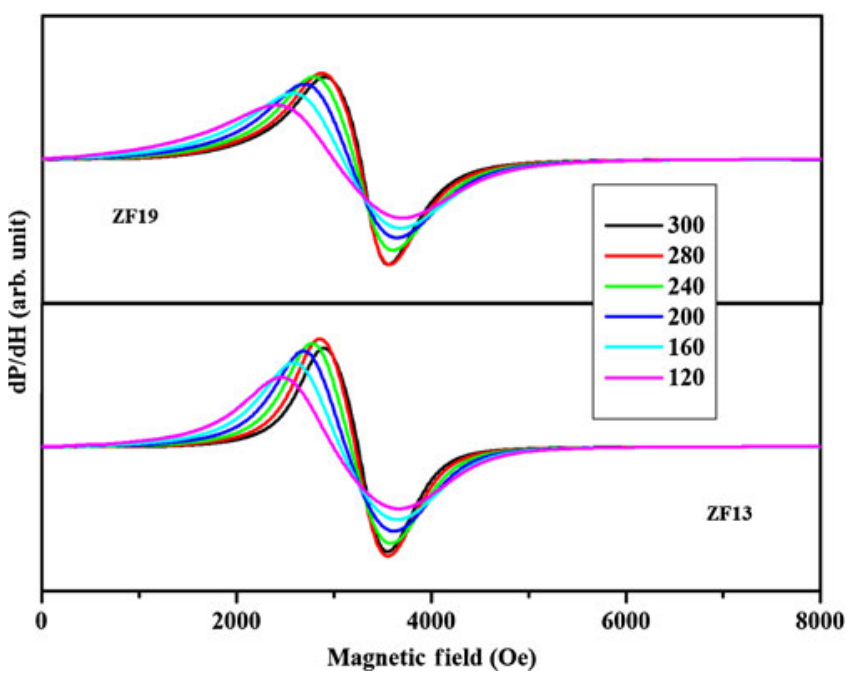

Figure 2. X-band EPR spectra of samples ZF13 and ZF19 at different temperatures. 
Table 1. Peak-to-peak linewidth $\left(\Delta H_{\mathrm{PP}}\right)$ and $g$-value of samples ZF13 and ZF19.

\begin{tabular}{lccccc}
\hline \multirow{2}{*}{$\begin{array}{l}\text { Temperature } \\
(\mathrm{K})\end{array}$} & \multicolumn{2}{c}{$\mathrm{ZF13}$} & & \multicolumn{2}{c}{$\mathrm{ZF19}$} \\
\cline { 2 - 3 } \cline { 5 - 6 } 120 & 1219 & $2 \cdot 253$ & & 1294 & $2 \cdot 254$ \\
160 & 1068 & $2 \cdot 198$ & & 1096 & $2 \cdot 186$ \\
200 & 938 & $2 \cdot 151$ & & 938 & $2 \cdot 141$ \\
240 & 820 & $2 \cdot 123$ & & 820 & $2 \cdot 107$ \\
280 & 688 & $2 \cdot 093$ & & 688 & $2 \cdot 077$ \\
300 & 649 & $2 \cdot 089$ & & 645 & $2 \cdot 066$ \\
\hline
\end{tabular}

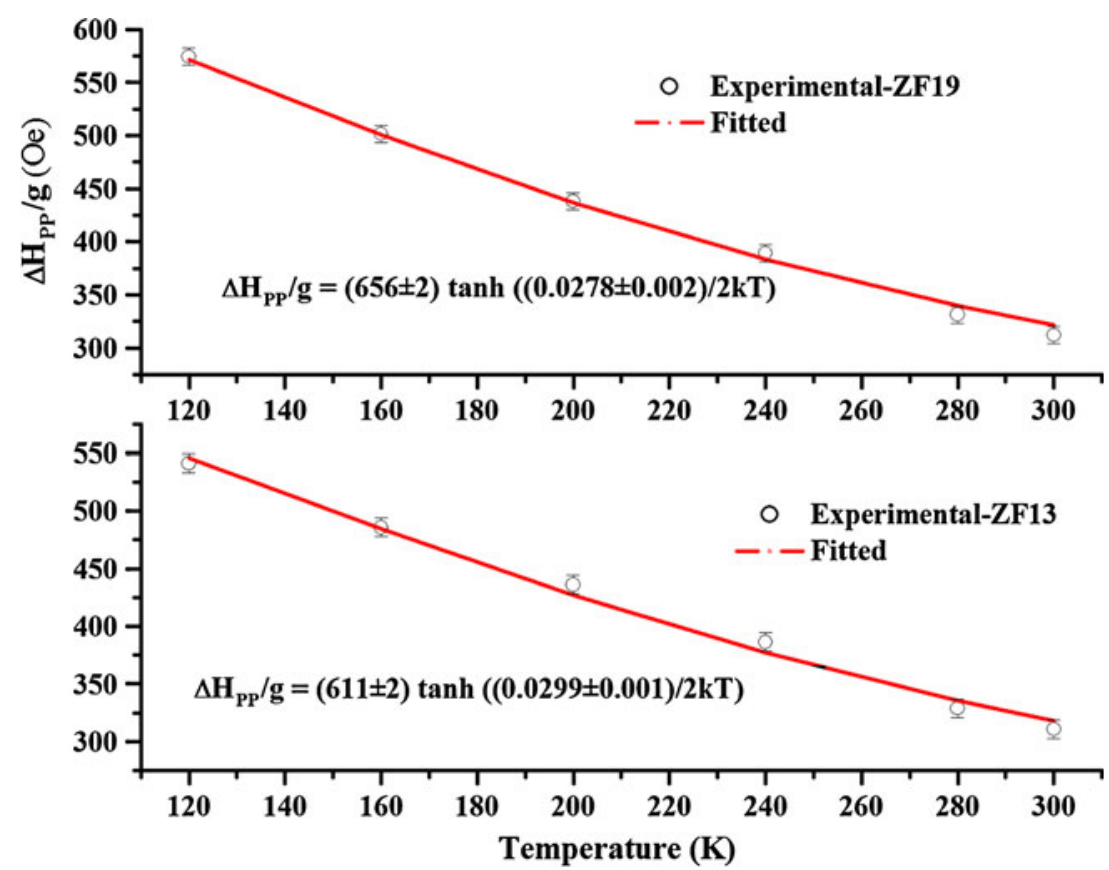

Figure 3. Variation of $\Delta H_{\mathrm{PP}} / g$ with temperature (blank circles show data points and red line is fitting to these points).

given by the following relation and may be applied to zinc ferrite (Wu et al 2004; Singh et al 2011)

$$
\frac{\Delta H_{\mathrm{PP}}}{g}=\frac{5 \beta S n}{R^{3}} \tan h\left(\frac{\Delta E}{2 k T}\right),
$$

where $\Delta E$ is the activation energy, $n$ the total number of magnetic centres, $R$ the average particle distance and $S$ the effective spins of magnetic centres. According to this equation, $\Delta H_{\mathrm{PP}} / g$ as a function of temperature has been plotted. Assuming $S, n$ and $R$ almost constant within the measured temperature range, data points were fitted by the above equation (figure 3). Fitting shows change in slope of the data points for both the samples. The values of $\Delta E$ obtained after fitting are $0.0299 \pm 0.001$ and $0.0278 \pm 0.001 \mathrm{eV}$ for samples ZF13 and ZF19, respectively. The values corresponding to factor $5 \beta S n / R^{3}$ are $611 \pm 2$ and $656 \pm 2$ for the samples ZF13 and ZF19. The differences in these values and slope may be related with the various factors that generally occur in nanoferrites with changes in crystallite size. These factors are different values of cation inversion and spin canting in both the samples. A detailed study carried out by using in-field Mössbauer spectroscopy on zinc ferrite nanoparticles of similar crystallite size indicates different values of cation inversion and spin canting at different sites (Singh et al 2012).

\section{Conclusions}

Superparamagnetic zinc ferrite was synthesized by nitrate method. Synthesized samples exhibit superparamagnetism at room temperature. The value of magnetization is lower for the large crystallite-sized sample. EPR study performed for these samples indicate the dominancy of superexchange interaction at higher temperature, while dipolar interaction at low temperature. The difference in values of activation energy of spins for both the samples has been attributed to the different values of cation inversion and spin canting. 


\section{Acknowledgements}

One of the authors (JPS) is thankful to Dr (Mrs) Veena Singh, Indian Institute of Technology, Kanpur, for recording EPR spectra of the samples and to CSIR, New Delhi, for providing a SRF during the course of this investigation.

\section{References}

Ahn Y, Choi E J, Kim S, An D H, Kang K U, Lee B G, Baek K S and Oak H N 2002 J. Korean Phys. Soc. 41123

Azam A, Chaman M and Naqvi A H 2009 Int. J. Nanopart. 2383

Chinnasamy C N, Narayanasamy A, Ponpandian N, Chattopadhyaya K, Guerault H and Greneche J H 2000 J. Phys.: Condens. Matter 127795

Cullity B D 1971 Introduction to magnetic materials (New York: Addison Wesley)

Dixit G, Singh J P, Srivastava R C and Agrawal H M 2012 J. Magn. Magn. Mater. 324479

Hofmann M, Campbell S J, Ehrhardt H and Feyerherm R 2004 J. Mater. Sci. 395057

Jaydevan B, Tohji K and Nakatsuka K J 1994 Appl. Phys. A76 3625

Lee S H, Broholm C, Ratclife W, Gasparovic G, Huang Q, Kim T H and Cheong S-W 2002 Nature Mater. 418856

Li L, Li G, Smith R L and Inomata H 2000 Chem. Mater. 123705

Nakashima S, Fujita K, Tanaka K, Hirao K, Yamamoto T and Tanaka I 2007 Phys. Rev. B75 17443

Poole C P and Farach H A 1971 Relaxation in magnetic resonance (London: Academic Press)
Roy M K, Halder B and Verma H C 2006 Nanotechnology 17 232

Sato T, Haneda K, Seki M and Iijima T 1990 Appl. Phys. A50 13

Shenoy S D, Joy P A and Anarantharaman M R 2004 J. Magn. Magn. Mater. 269217

Shim J H, Lee S, Park J H, Han S J, Jeong Y H and Cho Y W 2006 Phys. Rev. B73 064404

Singh J P, Srivastava R C, Agrawal H M, Kushwaha R P S, Chand $\mathrm{P}$ and Kumar R 2008a Int. J. Nanosci. 721

Singh J P, Srivastava R C, Agrawal H M and Kushwaha R P S 2008b Hyperfine Int. 183221

Singh J P, Srivastava R C, Agrawal H M and Chand P 2009 Int. J. Nanosci. 8523

Singh J P, Srivastava R C, Agrawal H M, Kumar R, Reddy V R and Gupta A 2010 J. Magn. Magn. Mater. 3221422

Singh J P, Srivastava R C, Agrawal H M, Chand P and Kumar R 2011 Curr. Appl. Phys. 11532

Singh J P, Dixit G, Srivastava R C, Agrawal H M, Reddy V R and Gupta A 2012 J. Magn. Magn. Mater. 3242553

Singh J P, Dixit G, Srivastava R C, Agrawal H M and Kumar R 2013 J. Alloys Compd. 551370

Singh S, Srivastava R C, Chand P, Khan D C and Wigner P E 1993 Solid State Commun. 8545

Sultan M and Singh R 2010 J. Phys.: Conf. Ser. 200722090

Wu K H, Chang Y C, Chen H B, Yang C C and Horng D N 2004 J. Magn. Magn. Mater. 278156

Xue H, Li Z, Wang X and Fu X 2007 Mater. Lett. 61347

Yao C et al 2007 J. Phys. Chem. C111 12274

Yu S H, Fujino T and Yoshimura M 2003 J. Magn. Magn. Mater. 256420 